\title{
Improvements of heat resistance and adhesive property of condensed poly-nuclear aromatic resin via epoxy resin modification
}

\author{
Wu Mingbo ${ }^{1 *}$, Wang Yuwei ${ }^{1,2}$, Jiang Wei ${ }^{1}$, Li Shibin ${ }^{1}$, Sun Qiqian ${ }^{1}$, Zheng \\ Jingtang $^{1}$ and Qiu Jieshan ${ }^{2 * *}$ \\ ${ }^{1}$ State Key Laboratory of Heavy Oil Processing, China University of Petroleum, Qingdao, Shandong 266580, China \\ ${ }^{2}$ Carbon Research Laboratory, State Key Lab of Fine Chemicals, School of Chemical Engineering, Dalian University of \\ Technology, Dalian 116024, China
}

(C) China University of Petroleum (Beijing) and Springer-Verlag Berlin Heidelberg 2014

\begin{abstract}
A bisphenol epoxy resin was used as modifier to increase the heat resistance of condensed poly-nuclear aromatic (COPNA) resin. The basic properties of COPNA resin and modified resin were characterized by Fourier transform infrared spectroscopy (FT-IR), nuclear magnetic resonance spectroscopy $\left({ }^{1} \mathrm{H}-\mathrm{NMR}\right)$, vapor pressure osmometry (VPO) and elemental analysis (EA). Average structural parameters of resins were calculated by the improved Brown-Ladner method, and heat resistance of resins was tested by thermogravimetric analysis (TGA). The chemical structure, mechanical properties and heat resistivity of the resin/graphite composites prepared with different resins were compared. The results show that the adhesive property and heat resistance of COPNA resin can be remarkably improved by addition of $5 \mathrm{wt} . \%$ epoxy resin. The reason is that the reactions between epoxy groups of epoxy resin and hydroxyl groups of COPNA resin improve the heat resistance and adhesive property of COPNA resin. Electric motor brushes with good mechanical properties and low electrical resistivity were successfully prepared by using the modified resin as binder.
\end{abstract}

Key words: COPNA resin, modification, epoxy resin, heat resistance, mechanical property

\section{Introduction}

COPNA resin, firstly reported in 1986 (Otani, 1986), has attracted considerable attention as a novel thermosetting material (Lin et al, 2010a; 2010b; Tanemura et al, 2011; 2012; $\mathrm{Wu}$ et al, 2012a; 2012b; 2013). It can be used as binder with good lubricity, mechanical properties and mouldability. In the early stages, the raw materials for preparation of COPNA resin were confined to pure aromatic substances (Nakajima et al, 1995; Nawa, 1996). Recently, petroleum- and coal-derived fractions have been employed to prepare COPNA resin with the aim of reducing costs (Guo et al, 2002; Li et al, 2008; Shi et al, 2012; Wu et al, 2012a; 2012b; 2013).

In our previous work, COPNA resin with good adhesive property was successfully synthesized via the reaction between thermally treated oil and 1, 4-benzenedimethanol ( $\mathrm{Wu}$ et al, 2013). However, the thermal decomposition temperature of the COPNA resin cured at $200{ }^{\circ} \mathrm{C}$ was still below $250{ }^{\circ} \mathrm{C}$, far low of that needed for industrial applications. To obtain resin with better heat resistance, epoxy resin was added

\footnotetext{
*Corresponding author. email: wumb@upc.edu.cn

**Corresponding author. email: jqiu@dlut.edu.cn

Received April 14, 2014
}

to the COPNA resin as a modifier, which was supposed to react with hydroxyl groups in COPNA resin. The resultant modified resin as well as COPNA resin was characterized by elemental analysis (EA), infrared spectroscopy (FT-IR), nuclear magnetic resonance spectroscopy $\left({ }^{1} \mathrm{H}-\mathrm{NMR}\right)$, vapor pressure osmometry (VPO) and thermogravimetric analysis (TGA). Finally, the modified COPNA resins as binder were mixed with graphite to prepare electric motor brushes, and their mechanical properties and electrical resistivity were studied.

\section{Experimental}

\subsection{Raw materials}

Toluene was obtained from Tianjin Chemical Reagent Company, China. Quinoline was bought from Tingxin Chemical Reagent Company, China. Bisphenol epoxy resin (E44) was purchased from Wuxi Resin Company, China. Chloroform was bought from Concord Technology Company, China. COPNA resin was synthesized from thermally treated oil and 1,4-benzenedimethanol as cross-linking agent at 150 ${ }^{\circ} \mathrm{C}$ in our lab (Wu et al, 2013). All reagents used in this work were of analytical grade. 


\subsection{Preparation of modified resin}

Epoxy resin was heated to $110^{\circ} \mathrm{C}$ and kept in a vacuum oven for $20 \mathrm{~min}$ to remove water. COPNA resin (50.0 g) was put in a reaction kettle equipped with a thermometer and mechanical stirrer and heated to $150{ }^{\circ} \mathrm{C}$ in a nitrogen flow of $40 \mathrm{~mL} / \mathrm{min}$, and then the above epoxy resin was rapidly added into the kettle just after the COPNA resin had completely melted and the mixture was stirred for $10 \mathrm{~min}$. Finally, the modified COPNA resin was obtained.

\subsection{Curing process of resins}

COPNA resin (10.0 g) and modified COPNA resin (10.0 g) were separately put in crucibles. The crucibles were heated to $200{ }^{\circ} \mathrm{C}$ at a heating rate of $0.25{ }^{\circ} \mathrm{C} / \mathrm{min}$ under a nitrogen flow of $50 \mathrm{~mL} / \mathrm{min}$, and then kept for $2 \mathrm{~h}$. Finally, the infusible and insoluble cured resins were obtained.

\subsection{Preparation of resin/graphite composite}

COPNA resin or modified resin $(10.0 \mathrm{~g})$ was dissolved in chloroform, and then graphite $(30.0,23.3,18.6$, or 15.0 g) was added into the solution. The mixture was stirred and heated to $80{ }^{\circ} \mathrm{C}$, until all the chloroform had evaporated. Then the resin/graphite composite was molded at $30 \mathrm{MPa}$ and 120 ${ }^{\circ} \mathrm{C}$. After that, the molded sample was heated to $300{ }^{\circ} \mathrm{C}$ at a heating rate of $0.25^{\circ} \mathrm{C} / \mathrm{min}$ under a nitrogen flow of $50 \mathrm{~mL} /$ min, and then kept for $2 \mathrm{~h}$.

\subsection{Characterization}

The modified resin and the COPNA resin were characterized by FT-IR, ${ }^{1} \mathrm{H}-\mathrm{NMR}$, EA, VPO, TGA, etc.

In thermogravimetric analysis (TGA), $10 \mathrm{mg}$ sample was placed in a platinum crucible of a DTG-60 thermogravimetric analyzer (Shimadzu, Japan), and heated from room temperature to $800{ }^{\circ} \mathrm{C}$ at a heating rate of $10{ }^{\circ} \mathrm{C} / \mathrm{min}$ under a nitrogen flow of $50 \mathrm{~mL} / \mathrm{min}$.

FT-IR spectra were recorded on a $8400 \mathrm{~S}$ FT-IR spectrometer (Shimadzu, Japan) in transmittance mode. The scan frequency of each spectrum was $15 \mathrm{~s}^{-1}$ with a resolution of $0.85 \mathrm{~cm}^{-1}$.

An AV500 ${ }^{1} \mathrm{H}-\mathrm{NMR}$ spectroscopy analyzer (Bruker, German) was used to characterize the state of hydrogen in the modified resin and the COPNA resin, with chloroform as solvent, trimethylphenylsilane as an internal standard, a sweep length of $10,000 \mathrm{~Hz}$, and a resonance frequency of 500 $\mathrm{MHz}$.

Elemental analysis (EA) was performed with an Element Vario EL organic element analyzer (Elementar, German). The structural parameters of the modified resin were calculated by the improved Brown-Ladner method (Boucher et al, 1990).

The VPO molecular weight of the resins was determined at $40{ }^{\circ} \mathrm{C}$ with a $\mathrm{K}-7000$ vapor pressure osmometer (Knauer, German), with chloroform as solvent, and dibenzoyl as a standard.

The contents of ash, toluene insolubles (TI) and quinoline insolubles (QI) were determined according to the international standards ISO 6245-1982, ISO 6376-1996, and ISO 6791-1981, respectively. The softening point of resins was determined according to ASTM D36-66.
Mechanical properties of resin/graphite composites were also studied. The flexural and compressive strengths of the composites were examined with a WDW-G universal material testing machine (Tenson, China), hardness was examined with a HS-19GDV scleroscope (Yuke, China), and electrical resistivity was determined according to JB/T8133-2-1999.

\section{Results and discussion}

\subsection{Basic properties of resins}

Epoxy resin has epoxy groups (as shown in Fig. 1), which are active and can be cross-linked to COPNA resin. The cured COPNA resin is insoluble and infusible, but its heat resistance does not meet the requirement of industrial applications, and needs to be improved. Fig. 1 shows the influence of epoxy resin content on the residual carbon yield of modified resin after it was kept in air at $500{ }^{\circ} \mathrm{C}$ for $2 \mathrm{~h}$. It can be seen that the residual carbon yield increased at first and then decreased with increasing epoxy resin. The maximum of residual carbon yield was at $5 \mathrm{wt} \%$ of epoxy resin. In the following discussion, the content of epoxy resin is all $5 \mathrm{wt} \%$.

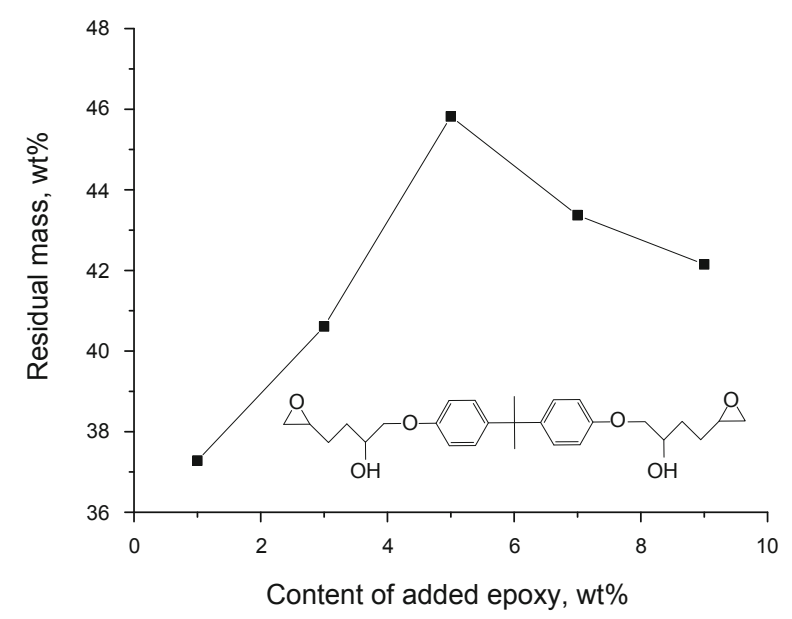

Fig. 1 Influence of epoxy resin content on the residual carbon yield of modified resin

Table 1 shows the basic properties of COPNA resin and modified COPNA resin with $5 \mathrm{wt} \%$ epoxy resin. It can be seen that after modification with $5 \mathrm{wt} \%$ of epoxy resin, the average molecular weight of COPNA resin increased from 1,412 to 1,962 , the residual carbon yield increased from 32.6 wt $\%$ to $45.8 \mathrm{wt} \%$, and the TI and QI values all increased. The value of TI-QI, the key parameter determining binding property, increased significantly from $12.4 \mathrm{wt} \%$ of COPNA resin to $20.3 \mathrm{wt} \%$ of modified COPNA resin. The above results indicated that the modified resin with high residual carbon yield and high TI-QI value is a more promising binder than the initial COPNA resin. From the EA data in Table 1, it is easily seen that the content of $\mathrm{O}$ is increased from 1.5 to 2.1 $w t \%$ after addition of epoxy resin.

\subsection{FT-IR analysis}

The FT-IR spectra of COPNA resin and modified resin are shown in Fig. 2. Peaks at $3,420-3,550 \mathrm{~cm}^{-1}$ were attributed to $\mathrm{N}-\mathrm{H}$ and $\mathrm{O}-\mathrm{H}$ stretching vibration. The larger peaks at 3,420- 
Table 1 Properties of COPNA resin and modified COPNA resin

\begin{tabular}{ccc}
\hline Properties & COPNA resin & Modified COPNA resin \\
\hline M & 1412 & 1962 \\
CR, wt.\% & 32.6 & 45.8 \\
QI, wt.\% & 5.6 & 6.4 \\
TI, wt.\% & 17.9 & 26.7 \\
(TI-QI), wt.\% & 12.4 & 20.3 \\
Softening point, ${ }^{\circ} \mathrm{C}$ & 110 & 112 \\
C, wt.\% & 89.1 & 88.9 \\
H, wt.\% & 6.1 & 7.0 \\
N, wt.\% & 0.4 & 0.3 \\
O, wt.\% & 1.5 & 2.1 \\
C/H & 1.22 & 1.06 \\
\hline
\end{tabular}

Notes: M, average molecular weight; CR, residual carbon ratio; QI, quinoline insoluble; TI, toluene insoluble

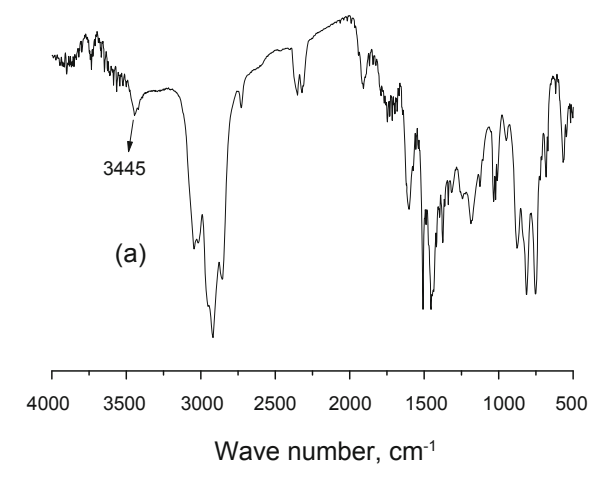

$3,550 \mathrm{~cm}^{-1}$ of modified resin compared with those of COPNA resin suggested more hydroxyl groups. The peaks at 1,378 and $1,360 \mathrm{~cm}^{-1}$ in Fig. 2 (b) were assigned to symmetrical dimethyl of epoxy resin. The peak of epoxy group at $910 \mathrm{~cm}^{-1}$ did not appear in both Fig. 2 (a) and (b), indicating that epoxy resin had reacted with COPNA resin and produced ether alcohol during the modification process.

\section{$3.3{ }^{1} \mathrm{H}-\mathrm{NMR}$ analysis}

Fig. 3 gives the ${ }^{1} \mathrm{H}-\mathrm{NMR}$ spectra of COPNA resin and modified resin. It was noteworthy that there were more peaks at $\delta 1.0-2.0$ in Fig. 3(b), indicating more hydrogen atoms of methyl groups in the modified resin. Peaks of modified resin at $83.5-5.0$ (Fig. 3(b)) were larger than those of COPNA resin (Fig. 3(a)), suggesting that more hydroxyl groups existed in modified resin. The large peaks at $\delta 6.0-9.0$ in Fig. 3(b) were attributed to the absorption resonance of hydrogen in aromatic rings. Peaks at $\delta 6.6-7.2, \delta 7.2-7.8$ and $\delta 7.8-8.3$ were assigned to hydrogen attached to simple, double and three

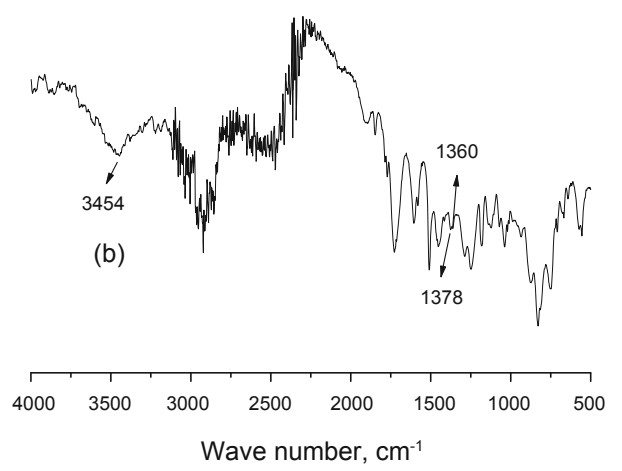

Fig. 2 FT-IR spectra of COPNA resin (a) and modified resin (b)
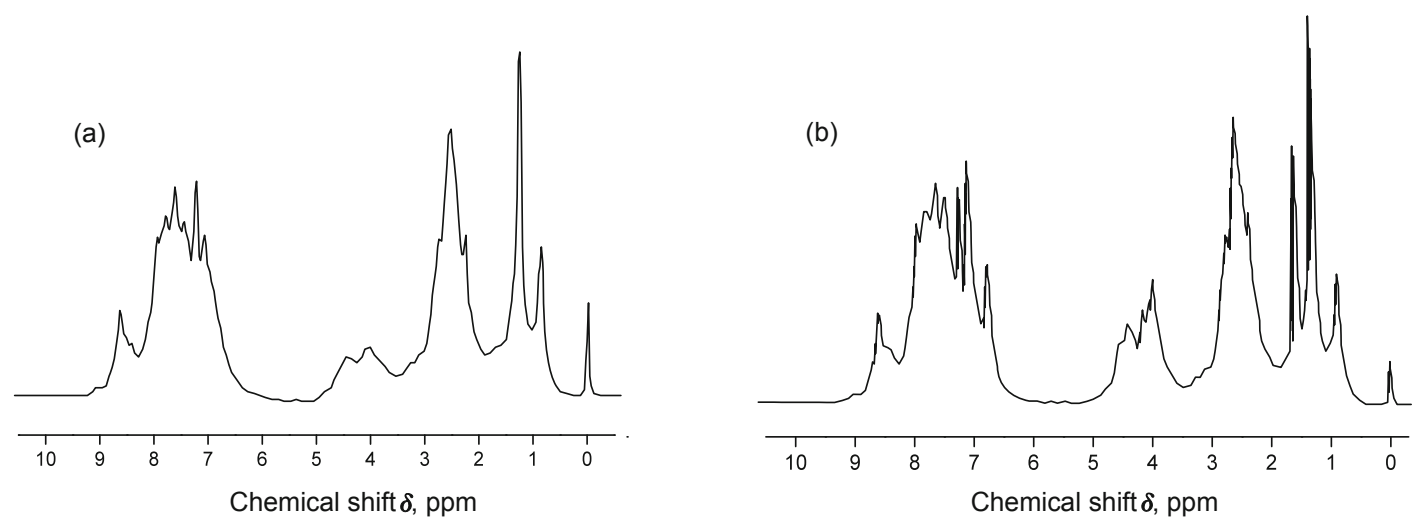

Fig. $3{ }^{1} \mathrm{H}-\mathrm{NMR}$ spectra of COPNA resin (a) and modified resin (b)

ring aromatics, respectively (Ahmaruzzaman et al, 2008; Molina V et al, 2010; Biswas et al, 2013). The peaks at $\delta 6.6-$ 7.2 were enhanced greatly in the modified resin due to more hydrogen atoms being attached to simple cyclic aromatics.

\subsection{Modification mechanism of COPNA resin using epoxy resin}

The average structural parameters of the COPNA and modified COPNA resins, including the average total carbon number $\left(\mathrm{C}_{\mathrm{T}}\right)$, the average total hydrogen number $\left(\mathrm{H}_{\mathrm{T}}\right)$, the ratio of aromatic carbon number to total carbon number $\left(f_{\mathrm{A}}\right)$, average condensation degree of aromatic rings $\left(\mathrm{H}_{\mathrm{AU}} /\right.$ $\mathrm{C}_{\mathrm{A}}$, atomic hydrogen to carbon ratio of the hypothetical unsubstituted aromatic molecule), hydrogen replacement rate surrounding aromatic rings $(\Sigma)$ and average total rings $\left(\mathrm{R}_{\mathrm{T}}\right)$, can be obtained by the improved Brown-Ladner method 
based on the results of FTIR, ${ }^{1} \mathrm{H}-\mathrm{NMR}$, EA and average molecular weight analysis. The average structural parameters of the resins are shown in Table 2, in which the S, O and $\mathrm{N}$ levels are negligible. The modification mechanism and averaged molecular structure of modified resin are shown in Fig. 4.

Table 2 Average molecular structural parameters of COPNA and modified resin

\begin{tabular}{ccccccc}
\hline Samples & $\mathrm{C}_{\mathrm{T}}$ & $\mathrm{H}_{\mathrm{T}}$ & $f_{\mathrm{A}}$ & $\mathrm{H}_{\mathrm{AU}} / \mathrm{C}_{\mathrm{A}}$ & $\Sigma$ & $\mathrm{R}_{\mathrm{T}}$ \\
\hline COPNA resin & 104.8 & 86.1 & 0.77 & 0.68 & 0.25 & 19.3 \\
Modified resin & 145.2 & 137.3 & 0.74 & 0.79 & 0.27 & 23.0 \\
\hline
\end{tabular}

As shown in Table 2, $\mathrm{R}_{\mathrm{T}}$ value increased from 19.3 of COPNA resin to 23.0 of modified resin, and $\mathrm{H}_{\mathrm{AU}} / \mathrm{C}_{\mathrm{A}}$ also increased remarkably, indicating the increase of polymerization degree after modification. Because the epoxy resin has more saturated carbon atoms, the value of $f_{\mathrm{A}}$ decreased from 0.77 to 0.74 . COPNA resin molecules were polymerized into large molecules through the reaction between hydroxyl groups and epoxy groups. When the hydroxyl groups in COPNA resin were completely reacted, the polymerization reactions between COPNA resin and epoxy resin ended. Excessive epoxy resin escaped at high temperature, resulting in the decrease of residual carbon yield of modified resin as shown in Fig. 1.

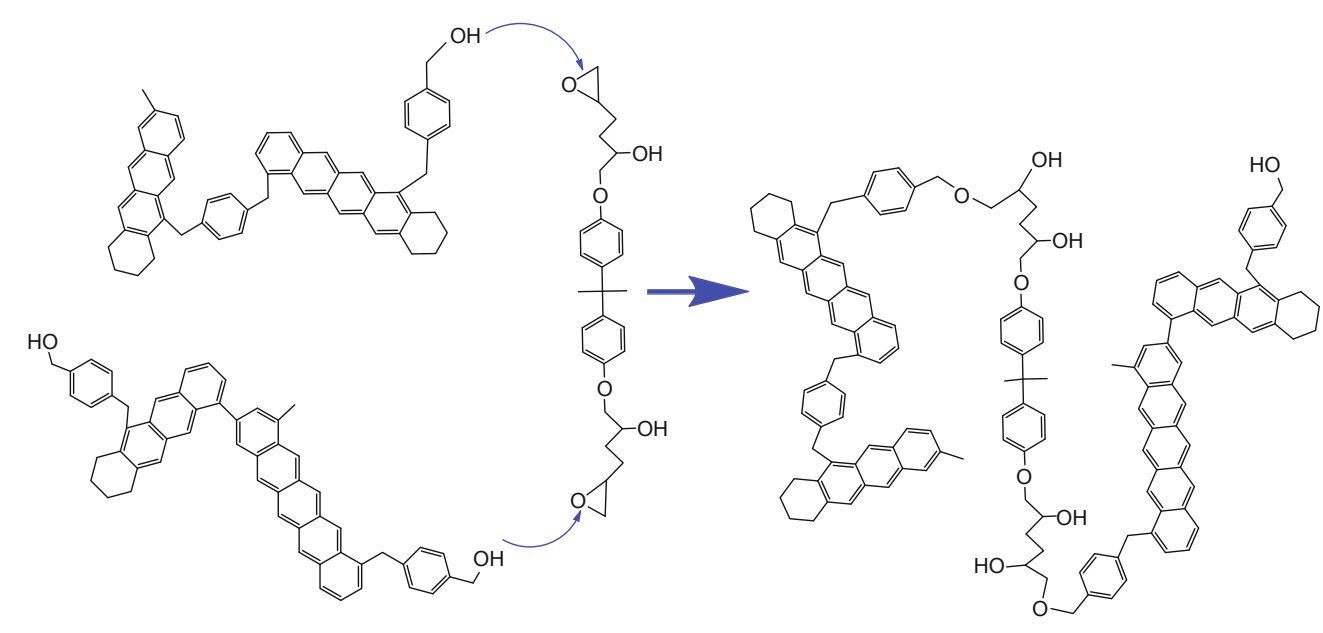

Fig. 4 Modification mechanism and averaged molecular structure of modified resin

\subsection{Heat resistance}

Fig. 5 shows the thermal decomposition behavior of cured COPNA resin and modified resin in nitrogen atmosphere. It can be clearly seen that the thermal decomposition temperature of cured COPNA resin was around $236{ }^{\circ} \mathrm{C}$, while that of cured modified resin was as high as $384{ }^{\circ} \mathrm{C}$. When the temperature was higher than $236{ }^{\circ} \mathrm{C}$, the light fraction of COPNA resin escaped and resulted in the significant weight loss. After modification, some light fraction of COPNA resin was connected with epoxy resin and polymerized into large molecules, resulting in the slow changing of the TG curve (Fig. 5(b)) as shown in Fig. 5. At $500{ }^{\circ} \mathrm{C}$, the residual weight of modified resin was still $47.1 \mathrm{wt} \%$, much higher than 30.7 $\mathrm{wt} \%$ of COPNA resin, indicating the cured modified resin has better heat resistance than cured COPNA resin. In other words, addition of a small amount of epoxy resin ( $5 \mathrm{wt} \%$ ) can effectively increase the heat resistance of cured COPNA resin. The high initial decomposition temperature and high TI-QI value indicated that modified resin was a good candidate for heat resistant binder.

\subsection{Mechanical properties of resin/graphite composite}

The mechanical properties and resistivity of cured composites are shown in Fig. 6. Fig. 6(a) and (b) showed that

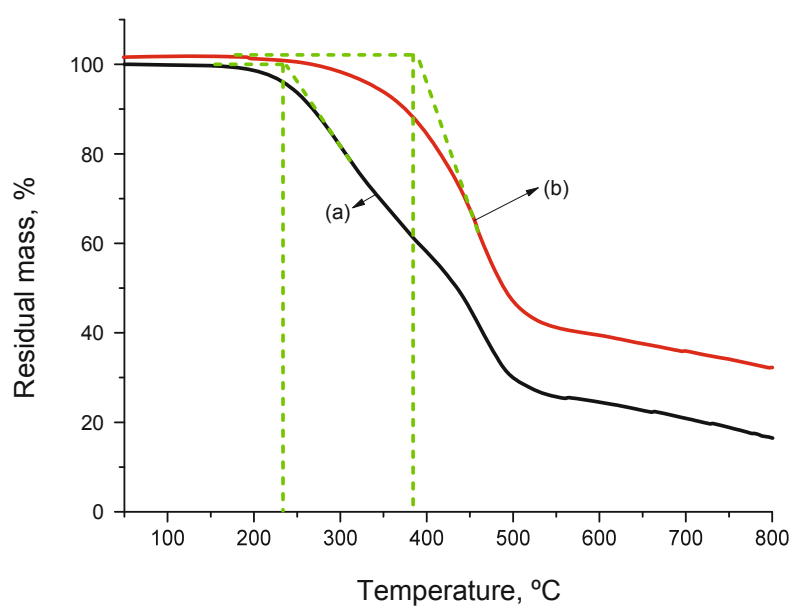

Fig. 5 TG curves of cured COPNA resin (a) and modified resin (b)

with increasing resin content from $25 \mathrm{wt} \%$ to $35 \mathrm{wt} \%$, the flexural and compressive strengths of both the composites increased, and the modified resin based composite possessed higher flexural and compressive strengths than COPNA resin based composite. When the resin content was higher than 35 $w t \%$, the flexural and compressive strengths of COPNA resin based composite decreased while those of modified resin based composite still increased. It is noted that the COPNA resin and modified resin can act as binder and contribute to 

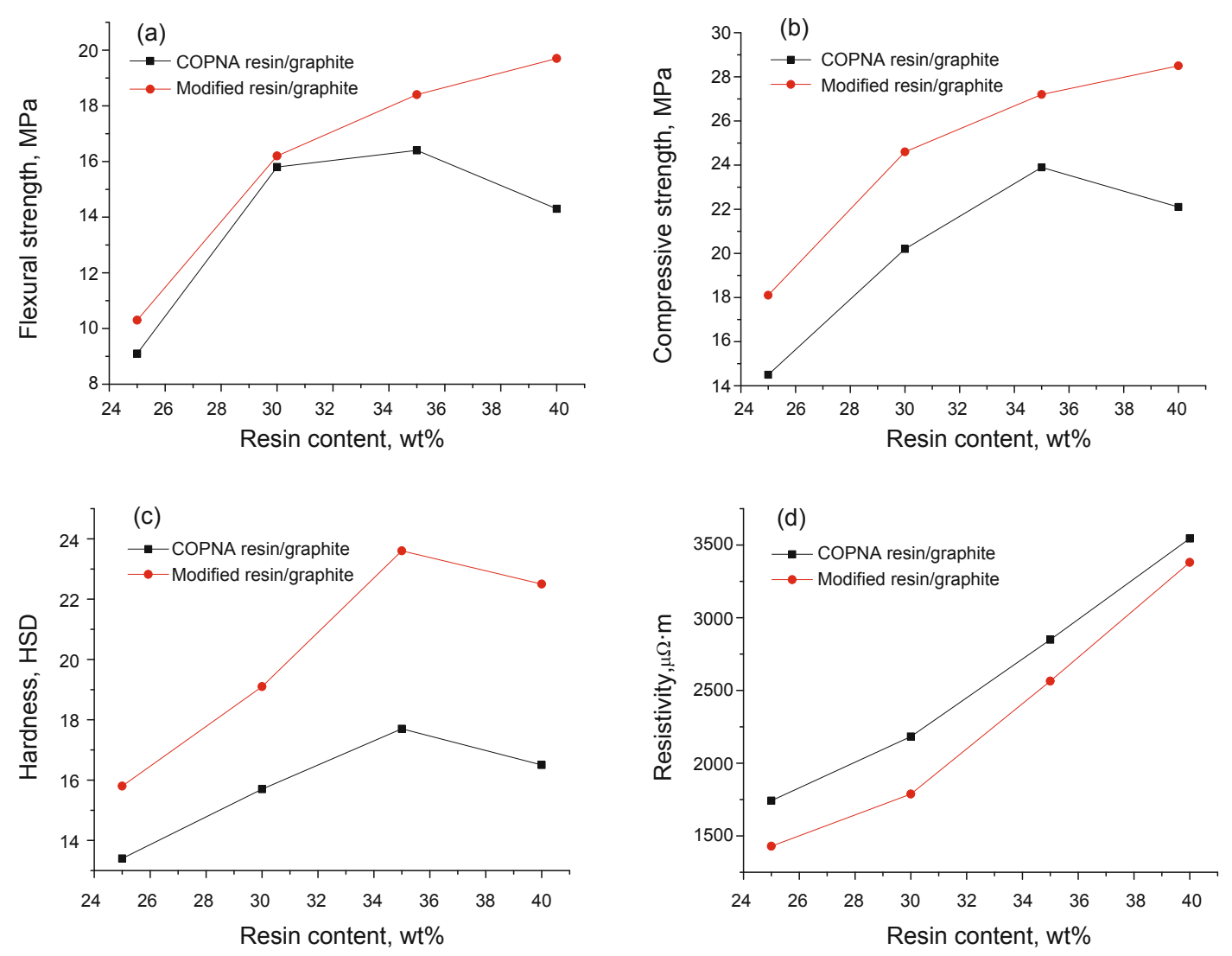

Fig. 6 Properties of cured composites: (a) flexural strength, (b) compression strength, (c) hardness, and (d) resistivity

the molding of the resin/graphite composite. However, when the COPNA resin was overabundant (higher than $35 \mathrm{wt} . \%$ ), the escape of low molecular weight elements in COPNA resin lowered the strength of the resin/graphite composite. The modified resin with $5 \mathrm{wt} \%$ epoxy resin had better heat resistance in than COPNA resin (Fig. 5), resulting in higher flexural and compressive strengths of modified resin based composite than that of COPNA resin based composite at high resin content. The hardness of COPNA resin based and modified resin based composites in Fig. 6(c) increased with increasing resin content, and achieved maximum at $35 \mathrm{wt} \%$ of resin content. The modified resin based composite was much harder than the COPNA resin based composite. Low resistivity is usually needed for resin as binder. Because the electrical resistivity of COPNA resin and modified resin was much higher than that of graphite, the resistivity of COPNA and modified resin based composites increased rapidly with increasing resin content (Fig. 6(d)), and the resistivity of the modified resin based composite was lower than that of the COPNA resin based composite, so it is suitable to be used as a binder.

\section{Conclusions}

A bisphenol epoxy resin was successfully used as a modifier to increase the performance of COPNA resin. The adhesive properties and heat resistance of COPNA resin were remarkably improved by addition of $5 \mathrm{wt} \%$ epoxy resin. This is very instructive for the application of COPNA resin as a binder at high temperature. The reaction between epoxy groups in added epoxy resin and hydroxyl groups in COPNA resin increases the heat resistance and adhesive properties of COPNA resin. Electric motor brushes made from modified resin/graphite composites exhibit good mechanical properties and low electrical resistivity, indicating potential future applications of the modified resin.

\section{Acknowledgments}

This work was supported by the National Natural Science Foundation of China (Nos. 51172285, 51372277); the Fundamental Research Funds for the Central Universities (14CX02060A); and the Natural Science Foundation of Shandong Province (ZR2011EL030).

\section{References}

Ahmaruzzaman M and Sharma D K. Characterization of liquid products from the co-cracking of ternary and quaternary mixture of petroleum vacuum residue, polypropylene, Samla coal and Calotropis Procera. Fuel. 2008. 87(10/11): 1967-1973

Biswas S, Mohanty P and Sharma D K. Studies on synergism in the cracking and co-cracking of Jatropha oil, vacuum residue and high density polyethylene: Kinetic analysis. Fuel Processing Technology. 2013. 106: 673-683

Boucher R J, Standen G, Patience R L, et al. Molecular characterization of kerogen from the Kimmeridge Clay Formation by mild selective chemical degradation and solid state ${ }^{13} \mathrm{C}-\mathrm{NMR}$. Organic Geochemistry. 1990. 16(6): 951-958

Molina V D, Uribe U N and Murgich J. Correlations between SARA 
fractions and physicochemical properties with ${ }^{1} \mathrm{H}-\mathrm{NMR}$ spectra of vacuum residues from Colombian crude oils. Fuel. 2010. 89(1): 185192

Guo Y S, Zha Q F and Wu M B. Synthesis of condensed polynuclear aromatic resins from aromatics-enriched feedstocks. Carbon Techniques. 2002. 119(02): 10-14 (in Chinese)

Li S B, Song C G and Hou B H. Study of the synthesis and composite properties of condensed poly-nuclear aromatics resin using coal tar pitch as monomer and trioxane as cross-linking agent. Carbon. 2008. 136(4): 13-17 (in Chinese)

Lin Q L, Zheng R G and Tian P H. Preparation and characterization of BMI resin/graphite oxide nanocomposites. Polymer Testing. 2010a. 29(5): 537-543

Lin Q L, Zheng M Z, Qin T, et al. Preparation of solid carbon spheres by pyrolysis of allyl COPNA-BMI resin. Journal of Analytical and Applied Pyrolysis. 2010b. 89(1): 112-116

Nakajima S, Iijima Y, Inoue T, et al. Anomalous magnetic properties of poly-condensate of fused polynuclear aromatics (COPNA) resin. Synthetic Metals. 1995. 71(1): 1817-1818

Nawa K. Development of flexible polymer blend films from advanced COPNA resin and Nylon 6. Journal of Applied Polymer Science. 1996. 61(10): 1737-1746

Otani S, Ota L and Raskovie V. Carbonization behavior of condensed poly-nuclear aromatic (COPNA) resins. Carbon (In Japanese). 1986. 127: $162-170$

Shi Y Y, Wu M B, Li S B, et al. Effects of cross-linking agents on synthesis of heat-resistant resin from ethylene tar. Applied Mechanics and Materials. 2012. 217-219: 1159-1165

Tanemura K, Suzuki T, Nishida Y, et al. Synthesis of the sulfonated condensed polynuclear aromatic (S-COPNA) resins as strong protonic acids. Tetrahedron. 2011. 67(6): 1314-1319

Tanemura K, Suzuki T, Nishida Y, et al. Synthesis of the strongly acidic sulfonated condensed polynuclear aromatic (S-COPNA) resins using aromatic aldehydes as cross-linking agents. Polymer Bulletin. 2012. 68(3): 705-719

Wu M B, Shi Y Y, Li S B, et al. Synthesis and characterization of condensed poly-nuclear aromatic resin using tiroxane as crossinglinking agent. New Carbon Materials. 2012a. 27(6): 469-475

Wu M B, Shi Y Y, Li S B, et al. Synthesis and characterization of condensed poly-nuclear aromatic resin derived from ethylene tar. China Petroleum Processing and Petrochemical Technology. 2012b. 14(4): 21-26

Wu M B, Jiang W, Wang Y W, et al. Synthesis of condensed polynuclear aromatic resin from furfural extract oil of reduced-pressure route II. Petroleum Science. 2013. 10(4): 584-588

(Edited by Zhu Xiuqin) 